
\title{
25 Research Soure \\ The Temporal Relation Between Pain and Fatigue in Individuals Receiving Treatment for Chronic Musculoskeletal Pain
}

\section{Keiko Yamada}

McGill University

Heather Adams

University Centre for Research and Disability Halifax

Tamra Ellis

Centre for Rehabilitation and Health

Robyn Clark

Kootenay Health Services Nelson

Craig Sully

Kootenay Health Services Nelson

Christian Lariviere

Occupational Health and Safety Research Institute Robert-Sauvé Montreal

Michael J. L. Sullivan ( $\sim$ Michael.Sullivan@McGill.ca )

McGill University

\section{Research Article}

Keywords: pain, fatigue, musculoskeletal, rehabilitation

Posted Date: October 14th, 2021

DOl: https://doi.org/10.21203/rs.3.rs-958935/v1

License: (c) (i) This work is licensed under a Creative Commons Attribution 4.0 International License.

Read Full License

Version of Record: A version of this preprint was published at BMC Musculoskeletal Disorders on March 8th, 2022. See the published version at https://doi.org/10.1186/s12891-022-05162-7. 


\section{Abstract}

Objective: Numerous investigations have revealed significant relations between pain and fatigue in individuals with persistent pain conditions. However, the direction of influence between pain and fatigue remains unclear. Shortcomings of design and analytic approaches used in previous research limit the nature of conclusions that can be drawn about possible causal relations between pain and fatigue. The present study investigated the temporal relation between changes in pain and changes in fatigue in individuals with musculoskeletal pain enrolled in a 10-week behavioral activation intervention. On the basis of previous findings, it was hypothesized that analyses would yield support for a bi-directional relation between pain and fatigue.

Methods: The study sample consisted of 104 individuals with chronic musculoskeletal pain participating in a 10-week standardized rehabilitation intervention. Measures of pain intensity and fatigue were completed pre-, mid-, and post-treatment. The three-wave data panel permitted examination of the direction of influence between pain and fatigue through the course of the intervention. A random-intercept cross-lagged panel model (RI-CLPM) was used to examine the temporal relation between pain and fatigue.

Results: Consistent with previous research, cross-sectional analyses of pre-treatment data revealed significant correlations between measures of pain and fatigue. Significant reductions in pain and fatigue were observed through the course of treatment ( $d=.33$ and $d=.66, \mathrm{p}<.001$, respectively). RI-CLPM revealed that pain severity predicted later fatigue (pre to mid-treatment standardized path coefficient $(\beta)=$ $0.55, p=0.02$; mid to post-treatment $\beta=0.36, p=0.001$ ); however, fatigue did not predict later pain severity.

Conclusions: Discussion addresses the processes that might underlie the temporal relation between pain and fatigue. Clinical implications of the findings are also discussed.

\section{Impact Statement}

The results of the study suggest that interventions that yield reductions in pain can also contribute to reduction in fatigue.

\section{Background}

Persistent fatigue is a frequent complaint of individuals with chronic pain [1]. Persistent fatigue has been broadly defined as overwhelming sense of tiredness, lack of energy and a feeling of exhaustion that is unrelated to the recent activity [2]. A relation between pain intensity and persistent fatigue has been reported in several populations where pain is a significant symptom including osteoarthritis [3], rheumatoid arthritis [4], fibromyalgia [5], cancer [6], headaches [7], and low back pain [8]. Approximately half of individuals with chronic pain report fatigue as their most debilitating symptom [2, 9]. 
Research conducted to date suggests that there might be a temporal relation between pain and fatigue [10]. Fishbain et al (1) reported that in 5 of the 6 prospective studies they reviewed, the development of fatigue occurred after pain onset, suggesting that pain might be causally related to fatigue. There are also indications that symptoms of fatigue might precede the onset of pain. Siivola et al [11] reported that symptoms of fatigue were prospectively associated with the onset of musculoskeletal pain in a sample of healthy young adults. Halder et al [12] reported that symptoms of fatigue were prospectively associated with the onset of abdominal pain in a sample of patients drawn from a primary care registry.

The pattern of findings that has emerged from previous research supports a relation between pain and fatigue but is limited in its implications for possible causal relations between pain and fatigue.

Demonstrating a prospective relation between pain and fatigue does not necessarily point to causal mechanisms. Prospective correlations can be influenced by extraneous sources of variance from autocorrelations (i.e., associations between the same variable measured at different times) and synchronous correlations (i.e., associations between different variables measured at the same time).

Analytic approaches that have been used in previous research, such as regression analyses, cross-lagged panel or path analytic procedures, account only for group-level changes between variables. Research suggests that failing to account for 'individual-level' changes in temporally-related variables increases the probability of Type I error, and consequently, can lead to erroneous conclusions [13]. It has been suggested that causal relations between temporally-related variables can best be addressed by analytic procedures that account for individual-level changes in variables of interest in addition to group-level changes [14].

In the present study, a random-intercept cross-lagged panel model (RI-CLPM) was used to examine the temporal relation between pain and fatigue in individuals with musculoskeletal conditions who were enrolled in a standardized rehabilitation intervention [14]. RI-CLPM is an extension of traditional CLPM permitting isolation of variance attributable to individual-level change [14]. Like traditional CLPM, RICLPM also controls for the potential inflation effects of auto- and synchronous relations among variables. RI-CLPM is considered a preferred analysis particularly in cases where variables might be expected to show trait-like stability, such as would be the case with chronic pain and associated fatigue [14]. Based on previous research, it was hypothesized that the results of analyses would support a bidirectional relation between pain and fatigue.

\section{Methods}

\section{Participants}

The study sample consisted of 104 consecutive referrals (41 men, 63 women) to an occupational rehabilitation service in Ontario, Canada. Selection criteria included a diagnosis of musculoskeletal pain involving the back or neck. The mean age of the sample was 44.9 years with a range of 29 to 61 years. The majority of the participants (62.5\%) were married or co-habiting and had completed at least 12 years 
of schooling (98.1\%). All participants were receiving wage indemnity from a work-injury or disability insurer.

\section{Measures}

Pain Severity. The McGill Pain Questionnaire Short-Form (MPQ-SF) was used to measure pain severity (Melzack, 1987). Participants rated their current pain experience according to 11 sensory and 4 affective pain descriptors on an intensity scale with the anchors (0) none, (1) mild, (2) moderate, or (3) severe. Scores can range from 0 to 45 where higher scores represent more intense pain. The measure has been shown to be reliable and valid in various clinical populations $[15,16]$.

Fatigue. The Fatigue Measure (FM) was used to assess fatigue [17]. The FM consists of 5 items assessing different symptoms of persistent fatigue. Ratings are made on a 3-point frequency scale with the anchors (0) never, (1) sometimes and (2) often. Scores on the measure of fatigue can range from 0 and 10. Research has supported the reliability and validity of the FM in individuals with musculoskeletal pain [17]. In the present sample, Cronbach's alpha for the fatigue measure was .78.

\section{Procedure}

This study was approved by Institutional Review Board of McGill University. The study sample was drawn from the de-identified clinical files of individuals who were enrolled in a 10-week standardized risktargeted behavioural activation intervention (e.g., activity scheduling, goal setting, exposure to success and achievement experiences). The risk-targeted behavioural activation intervention in which participants were enrolled is described in more detail elsewhere [18]. Participation in risk-targeted behavioral activation has been associated with clinically significant reductions in pain and fatigue [19]. The intervention was offered as a complement to any other pharmacological or physical interventions that might have been prescribed for symptom management. All participants received treatment between January and December, 2017. Data were extracted in May of 2018.

Clinicians were occupational therapists who had been trained to competency in the risk-targeted behavioral activation intervention. All clinicians were supervised by a senior clinician to ensure fidelity to the standardized treatment protocol. Measures of pain severity and fatigue were completed pre-treatment (week 0), mid-treatment (week 4) and treatment termination (week 9).

\section{Data Analytic Approach}

T-tests for independent samples were used to compare women and men on continuous variables and chi square analyses were used to compare groups on categorical variables. Pearson correlations were used to examine relations among variables at time of admission, mid- and post-treatment. T-tests for paired variables were used to examine treatment-related changes in fatigue, pain, and depressive symptoms. Intra-class correlation (ICC) using a two-way random effects model was calculated for variables. ICC can be defined as the proportion of the variance explained by differences between participants. RI-CLPM was used to examine the temporal associations between treatment-related changes in symptoms of pain and 
fatigue following the methods introduced by Hamaker et al. [14]. One of the advantages of RI-CLPM over other approaches to time-series or path analyses is that RI-CLPM isolates within-person associations between two (or more) variables from between-person associations attributable to trait-like characteristics [20]. RI-CLPM allows parsing the part of the variability of a measure that is invariant, traitlike, from genuine autoregressive stability, which results in a more precise estimation of the cross-lagged relationships. Model fitting indices included the comparative fit index (CFI), the Tucker-Lewis index (TLI), the root mean square error of approximation (RMSEA) with 90\% confidence interval (Cl), and the standardized root-mean-square residual (SRMR) [21].

T-tests for paired variables and Pearson's correlations were conducted with SAS version 9.4 (SAS Institute Inc., Cary, NC, USA), ICC was calculated with IBM SPSS statistics version 24, and the RI-CLPM was conducted with M-Plus version 8.4. A portion of these data have been used in a previous publication addressing the relation between fatigue and occupational disability [19].

\section{Results}

\section{Sample Characteristics}

Means and standard deviations on all study variables are presented stratified by sex in Table 1. Median pain duration for the study sample was approximately 1 year. The majority of participants were using some form of analgesic for pain control. Approximately $10 \%$ of the sample was concurrently receiving another form of rehabilitation treatment. Based on test scores at the time of admission, the study sample would be characterized as experiencing moderate to severe symptoms of pain and fatigue [19]. 
Table 1

Sample Characteristics at the Time of Admission

\begin{tabular}{|c|c|c|c|c|c|}
\hline \multirow{3}{*}{ 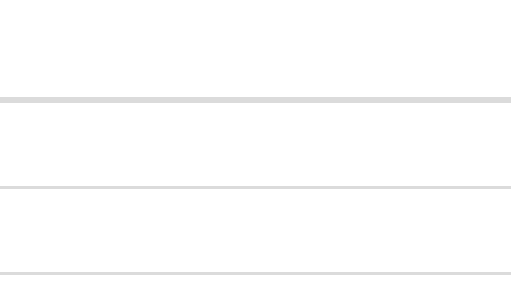 } & \multirow{2}{*}{\multicolumn{2}{|c|}{$\begin{array}{l}\text { Men } \\
N=41\end{array}$}} & \multirow{2}{*}{\multicolumn{2}{|c|}{$\begin{array}{l}\text { Women } \\
N=63\end{array}$}} & \multirow[b]{3}{*}{ P-value } \\
\hline & & & & & \\
\hline & Mean & SD & Mean & SD & \\
\hline \multirow[t]{2}{*}{ Age, years } & 44.7 & 8.0 & 45.1 & 8.0 & 0.83 \\
\hline & $\mathrm{n}$ & $\%$ & $\mathrm{n}$ & $\%$ & \\
\hline \multicolumn{6}{|l|}{ Marital Status } \\
\hline Single & 10 & $24.4 \%$ & 13 & $20.6 \%$ & 0.61 \\
\hline Married or co-habiting & 26 & $63.4 \%$ & 39 & $61.9 \%$ & 0.46 \\
\hline Separated or divorced & 4 & $9.8 \%$ & 10 & $15.9 \%$ & - \\
\hline Widowed & 1 & $2.3 \%$ & 1 & $1.6 \%$ & - \\
\hline \multicolumn{6}{|l|}{ Education } \\
\hline Less than high school & 2 & $4.9 \%$ & 0 & $0.0 \%$ & - \\
\hline High school/Trade school & 13 & $31.7 \%$ & 16 & $25.4 \%$ & 0.48 \\
\hline College & 13 & $31.7 \%$ & 37 & $58.7 \%$ & 0.007 \\
\hline University & 12 & $29.3 \%$ & 10 & $15.9 \%$ & 0.10 \\
\hline Missing & 1 & $2.4 \%$ & 0 & $0.0 \%$ & - \\
\hline Pain Duration & & & & & 0.88 \\
\hline $3-6$ months & 4 & $9.8 \%$ & 8 & $12.7 \%$ & \\
\hline $7-12$ months & 15 & $36.6 \%$ & 21 & $33.3 \%$ & \\
\hline More than 12 months & 22 & $53.7 \%$ & 34 & $54.0 \%$ & \\
\hline \multicolumn{6}{|l|}{ Medication } \\
\hline NSAIDs & 26 & $63.4 \%$ & 32 & $50.8 \%$ & 0.21 \\
\hline Opioid & 6 & $14.6 \%$ & 9 & $14.3 \%$ & 0.96 \\
\hline Antidepressant & 8 & $19.5 \%$ & 7 & $11.1 \%$ & 0.14 \\
\hline \multicolumn{6}{|l|}{ Secondary treatment } \\
\hline Physiotherapy & 3 & $7.3 \%$ & 1 & $1.6 \%$ & - \\
\hline
\end{tabular}

Note: $\mathrm{N}=104$. FM: Fatigue Measure, MPQ: McGill Pain Questionnaire - Short Form, NSAIDS: Nonsteroidal anti-inflammatory drug, SD: Standard Deviation. 


\begin{tabular}{|llllll|}
\hline \multicolumn{7}{|c|}{ Men } & \multicolumn{5}{l|}{ Women } \\
\hline Occupational therapy & 3 & $7.3 \%$ & 5 & $7.9 \%$ & - \\
\hline Psychotherapy & 0 & $0.0 \%$ & 1 & $1.6 \%$ & - \\
\hline MPQ-SF $(0-45)$ & Mean & SD & Mean & SD & \\
\hline FM $(0-10)$ & 17.6 & 8.7 & 19.0 & 9.2 & 0.40 \\
\hline $\begin{array}{l}\text { Note: N=104. FM: Fatigue Measure, MPQ: McGill Pain Questionnaire - Short Form, NSAIDS: } \\
\text { Nonsteroidal anti-inflammatory drug, SD: Standard Deviation. }\end{array}$ & \\
\hline
\end{tabular}

\section{Pain Severity and Fatigue: Cross-Sectional Relations}

Consistent with previous research [22], pain severity was significantly correlated with fatigue at admission (Table 2). Pain severity and fatigue were also significantly correlated at mid- and posttreatment, $r=.34, p<.001, r=.57, p<.001$, respectively.

Table 2

Zero-order Correlations Between Pain and Fatigue at Pre-, Mid-, and Post-Treatment

\begin{tabular}{|c|c|c|c|c|c|}
\hline & 1 & 2 & 3 & 4 & 5 \\
\hline $1 \mathrm{MPQ}-\mathrm{PRI} \mathrm{1}_{1}$ & - & - & - & - & - \\
\hline $2 \mathrm{MPQ}-\mathrm{PRI}{ }_{2}$ & $0.67^{\star \star \star}$ & - & - & - & - \\
\hline $3 \mathrm{MPQ}-\mathrm{PRI} \mathrm{I}_{3}$ & $0.47^{\star \star \star}$ & $0.70^{\star \star \star}$ & - & - & - \\
\hline $4 \mathrm{FM}_{1}$ & $0.39 * \star \star$ & $0.34^{\star \star \star}$ & $0.27^{\star \star}$ & - & - \\
\hline $5 \mathrm{FM}_{2}$ & $0.34^{\star \star \star}$ & $0.34^{\star \star \star *}$ & $0.33^{\star \star \star}$ & $0.54^{\star \star \star}$ & - \\
\hline $6 \mathrm{FM}_{3}$ & 0.21 * & $0.38^{* \star *}$ & $0.57^{\star \star *}$ & $0.50 * \star *$ & $0.59 * \star *$ \\
\hline \multicolumn{6}{|l|}{ Note: $\mathrm{N}=104$} \\
\hline \multicolumn{6}{|c|}{ FM; Fatigue Measure, MPQ-PRI; McGill Pain Questionnaire-Pain Rating Index. } \\
\hline \multicolumn{6}{|c|}{$\begin{array}{l}* p<0.05, * \star p<0.01,{ }^{* \star *} p<0.001 . \text { Subscripts: } 1=\text { pre-treatment, } 2=\text { mid-treatment, } 3=\text { post- } \\
\text { treatment. }\end{array}$} \\
\hline
\end{tabular}

\section{Treatment-Related Changes in Dependent Variables}

As shown on Table 3, t-tests for paired samples (pre- to mid-, mid- to post-, and pre- to post-treatment) were conducted to examine treatment-related changes in scores on measures of pain and fatigue 
symptom severity. Participation in the treatment program (pre- to post-treatment) was associated with significant reductions in pain severity, $\mathrm{t}(102)=3.3, \mathrm{p}<.001 . d=.33(95 \% \mathrm{Cl}, 0.13-0.52]$ and fatigue, $\mathrm{t}$ $(102)=6.7, \mathrm{p}<.001, d=.66(95 \% \mathrm{Cl} .0 .45-0.87)$.

Table 3

Means Scores for Pain and Fatigue at Pre-, Mid-, and Post-Treatment

\begin{tabular}{|c|c|c|c|c|}
\hline MPQ-PRI (0 - 45) & Pre-treatment & Mid-treatment & Post-treatment & \\
\hline Mean (SD) & $18.5(9.0)$ & $17.1(10.0)$ & $15.1(10.8)$ & \\
\hline \multicolumn{5}{|l|}{ pre- to mid- } \\
\hline$p$ value & & 0.07 & & \\
\hline Cohen's d $(95 \% \mathrm{Cl})$ & & $0.18(-0.01-0.38)$ & & \\
\hline \multicolumn{5}{|l|}{ mid- to post- } \\
\hline$p$ value & & & 0.02 & \\
\hline Cohen's d $(95 \% \mathrm{Cl})$ & & & $0.24(0.05-0.44)$ & \\
\hline \multicolumn{5}{|l|}{ pre- to post- } \\
\hline$p$ value & & & & $<0.001$ \\
\hline Cohen's d $(95 \% \mathrm{Cl})$ & & & & $0.33(0.13-0.52)$ \\
\hline FM $(0-10)$ & Pre-treatment & Mid-treatment & Post-treatment & \\
\hline Mean (SD) & $7.6(2.1)$ & $6.6(2.4)$ & $5.9(2.9)$ & \\
\hline \multicolumn{5}{|l|}{ pre- to mid- } \\
\hline$p$ value & & $<0.001$ & & \\
\hline Cohen's d $(95 \% \mathrm{Cl})$ & & $0.49(0.28-0.69)$ & & \\
\hline
\end{tabular}

mid- to post-

$p$ value

0.01

Cohen's d $(95 \% \mathrm{Cl})$

$0.26(0.06-0.45)$

pre- to post-

p value $<0.001$

Cohen's d $(95 \% \mathrm{Cl})$ $0.66(0.45-0.87)$

Note: $\mathrm{N}=104$.

Cl; Confidence Interval, FM; Fatigue Measure, MPQ-PRI; McGill Pain Questionnaire-Pain Rating Index, SD; standard deviation. 


\section{Random Intercept Cross-Lagged Panel Model (RI-CLPM) Between Pain and Fatigue}

The results of the RI-CLPM for the temporal association between pain and fatigue, for within- and between-person effects are presented in Figure 1, and standardized estimates of all paths in RI-CLPM are shown in Table 4. Variance and covariance of random-intercept MPQ-PRI were set to zero because the random-intercept for the MPQ-PRI was not significant. For fatigue, the intra-class correlation (ICC) was 0.53 (95\% Cl, 0.42-0.64); differences in fatigue between participants (i.e., between-person variance) explained $53 \%$ of the variance in fatigue across the three measurement points, and within-person variance explained the remaining $47 \%$ of the variance across measurement points.

Table 4

Random Intercepts Cross-Lagged Panel Path Coefficients

\begin{tabular}{llll}
\hline Parameter & $\boldsymbol{\beta}$ & SE & $\mathbf{p}$ \\
\hline Within-person (state-like) & & & \\
\hline Autoregressive paths & & & \\
\hline $\mathrm{MPQ}-\mathrm{PRI} 1 \rightarrow \mathrm{MPQ}-\mathrm{PRI} 2$ & 0.60 & 0.1 & $<0.001$ \\
\hline $\mathrm{MPQ}-\mathrm{PRI} 2 \rightarrow \mathrm{MPQ}-\mathrm{PRI} 3$ & 0.65 & 0.07 & $<0.001$ \\
\hline $\mathrm{FM} 1 \rightarrow \mathrm{FM} 2$ & -0.14 & 0.3 & 0.63 \\
\hline FM2 $\rightarrow$ FM3 & 0.23 & 0.1 & 0.08
\end{tabular}

\section{Correlations}

$\begin{array}{llll}\text { MPQ-PRI1 - FM1 } & 0.59 & 0.1 & <0.001 \\ \text { MPQ-PRI2 - FM2 } & 0.19 & 0.1 & 0.18 \\ \text { MPQ-PRI3 - FM3 } & 0.52 & 0.08 & <0.001\end{array}$

\section{Cross-lagged paths}

\begin{tabular}{llll} 
MPQ-PRI1 $\rightarrow$ FM2 & 0.55 & 0.2 & 0.02 \\
\hline MPQ-PRI2 $\rightarrow$ FM3 & 0.36 & 0.1 & 0.001 \\
FM1 $\rightarrow$ MPQ-PRI2 & 0.12 & 0.2 & 0.37 \\
FM2 $\rightarrow$ MPQ-PRI3 & 0.12 & 0.09 & 0.18
\end{tabular}

Note: $\mathrm{N}=104$.

$\beta$; standardized estimate, FM; Fatigue Measure, MPQ-PRI; McGill Pain Questionnaire-Pain Rating Index, SE; standard error. Random intercept cross-lagged panel model (RICLPM) was used to estimate path coefficient. Variance and covariance of random-intercepts for the MPQ-PRI were set to zero because random-intercepts of MPQ-PRI were not significant; there was no effect of between-person (trait-like) for the MPQ-PRI. 
There were significant cross-sectional associations between pain severity and fatigue symptoms at time of admission and post-treatment at a within-person level $(\beta=0.59, p<0.001$, and $\beta=0.52, p<0.001)$. The autoregressive paths for pain severity through the course of treatment were significant at pre- to midtreatment and mid to post-treatment $(\beta=0.60, p<0.001$ and $\beta=0.65, p<0.001$, respectively), indicating that within-person deviations of pain severity from expected scores (i.e., the mean) predicted deviations from expected scores at the next time point. On the other hand, the autoregressive paths for fatigue symptoms through the course of treatment at the within-person level were not significant. Within-person cross-lagged paths from pain severity to fatigue symptoms were significant at pre-and mid-treatment ( $\beta$ $=0.55, p=0.02)$ and mid- and post-treatment $(\beta=0.36, p=0.001)$; pain severity predicted fatigue symptoms. There were no significant cross-lagged effects from fatigue symptoms to pain severity; fatigue symptoms at pre- and mid-treatment did not predict pain severity at the next time point (mid-and post-treatment). The overall model fit of the RI-CLPM was good, CFI $=1.00, T L I=1.00, S R M R=0.03$, and RMSEA $=<0.001(90 \% \mathrm{Cl}, 0.00-0.15)$.

\section{Discussion}

The results of the study are consistent with previous research showing that scores on a measure of pain intensity are significantly correlated with scores on a measure of fatigue [1]. The findings of the present study extend previous research in showing that, through the course of a behavioral activation intervention, early treatment changes in pain predicted later changes in fatigue; but early treatment changes in fatigue did not predict later changes in pain intensity. The findings do not support a bidirectional relation between pain and fatigue. To our knowledge, this is the first study to show that, during a rehabilitation intervention, early changes in pain severity predict later changes in fatigue.

\section{Temporal Relation between Pain and Fatigue.}

The results of systematic reviews suggest that fatigue is a common symptom reported by individuals with persistent pain conditions $[1,23]$. Several prospective studies have reported that changes in pain precede changes in fatigue $[8,24,25]$. For example, Feuerstein et al [8] reported that changes in pain intensity were prospectively associated with changes in the severity of fatigue in patients with low back pain. Similar findings were reported by Nicassio et al [24] in a sample of patients with fibromyalgia. The findings of Feuerstein et al [8] and Nicassio et al [24] suggest that the functional linkage between pain and fatigue is proximal in time; in both studies, variations in pain significantly predicted next-day pain. Christie et al [26] examined the time-lagged relation between pain and fatigue in patients with rheumatoid arthritis over a one-week period. Their findings suggested that pain prospectively predicted later fatigue only in a subset of patients. For the majority of patients, the relation between pain and fatigue was synchronous.

Several different mechanisms have been proposed to account for a temporal relation between pain and fatigue. Resource depletion accounts have been proposed where it has been suggested that the physical 
or psychological energy required to manage or cope with persistent pain ultimately depletes energy resources contributing to the experience of fatigue [27]. Olson and colleagues have conceptualized fatigue as a component of the stress response [28]. Van Damme et al [10] suggest that pain-related fatigue is the subjective experience of disengagement from goal pursuits that occurs when the costs of pursuing activity goals exceed the benefits. The disruptive effects of pain on sleep have also been discussed as contributing to pain-related fatigue [29].

Research has also pointed to a number of physiological processes that might give rise to fatigue associated with pain. It has been suggested that systemic inflammation might underlie the association between pain and fatigue [30,31]. For example, the inflammatory phase accompanying musculoskeletal injuries of the spinal column induces proinflammatory cytokines implicated in the multifidus muscle remodeling. These changes can lead to muscle fatigue as replacement of muscle fibers by adipose tissues weakens the muscle and decreases muscle endurance [32]. Other potential mediators of the relation between pain and fatigue include reductions in heat shock protein (HSP), brain-derived neurotrophic factor (BDNF) [33] and lower dopamine levels [34].

\section{Temporal Relation between Fatigue and Pain.}

Fewer studies have provided support a prospective relation between fatigue and pain. Studies examining the trajectory of symptoms reported by primary care patients have revealed that symptoms of fatigue prospectively predict the later emergence of pain symptoms $[11,12]$. In a community health survey of high school students, Siivola et al [11] reported that participants who reported symptoms of fatigue were more likely to have developed a musculoskeletal pain condition when followed up 7 years later. Halder et al [12] reported that high scores on a measure of fatigue predicted onset of abdominal pain in a community sample of adults when re-assessed 1-year later. Aili et al [29] reported that symptoms of fatigue pre-dated the onset of pain in a large sample of individuals who later (5 years) developed chronic widespread pain.

Our failure to replicate previous findings showing a temporal relation between fatigue and pain might be due of several factors. First, the time frame of our study might not have been adequate to capture the time-dependent nature of the relation between fatigue (as a causal factor) and pain. Studies that have yielded findings supportive of a temporal relation between fatigue and pain have had follow-up periods ranging from 1 to 7 years. The timeframe of the present study extended only over a 10-week period. As well, studies reporting evidence of a temporal relation between fatigue and pain sought to determine the role of fatigue symptoms as a prognostic indicator of the onset of a pain condition at a later point in time. The present study addressed whether early treatment-related reductions in fatigue predicted later reductions in pain. It is possible that the mechanisms that underlie the prognostic value of fatigue for the onset of a future pain condition differ from those that are associated with treatment response.

It is important to consider that the analytic procedures used in all studies supporting a temporal relation between fatigue and pain have used group-based procedures, and have not controlled for within-person associations. In all studies, the analytic approach involved the use of logistic regressions using 
categorized groups of fatigue with a binary outcome (presence of pain or not) to calculate odds ratios. As noted earlier, group-based procedures are associated with a higher rate of Type I error. In support of this explanation, when the data from the present study were analyzed using traditional CLPM, which does not control for within-person variance, results supported a bi-directional relation between pain and fatigue. However, when analyzed using RI-CLPM procedure, controlling for within-person variance, the paths from fatigue to pain were no longer significant.

\section{Clinical Perspectives}

Pain is an important determinant of magnitude of disability associated with musculoskeletal conditions [35]. Emerging research suggests that fatigue is a symptom that frequently co-occurs with musculoskeletal pain, and further adds to the burden of disability [36, 37]. Indeed, research shows that treatment-related reductions in fatigue prospective predict resumption of occupational activities in individuals with musculoskeletal conditions [19]. Surprisingly, there is a paucity of research that has addressed how best to manage debilitating symptoms of fatigue to promote rehabilitation progress in individuals with musculoskeletal conditions.

In the present study, participation in a behavioral activation intervention was associated with a $20 \%$ reduction in fatigue, corresponding to a moderate effect size $(d=.66)$. The effect size was higher when analyses were conducted only on the subsample of participants who initially presented with severe (> $6 / 10)$ symptoms of fatigue $(d=.71)$. In previous research, reductions in fatigue of $20 \%$ (medium effect size) have been shown to be sufficient to translate into meaningful clinical outcomes such as return to work [19].

The pattern of findings of the present study suggests that early-treatment reductions in pain might contribute to reductions in fatigue in the rehabilitation of musculoskeletal injury. In turn, reductions in fatigue might promote fuller engagement in treatment, and contribute to more positive rehabilitation outcomes [19]. The data also suggest that only minor reductions in pain severity were required to achieve meaningful reductions in fatigue. It is not clear however, that all techniques, modalities or medications that reduce pain severity will also be associated with reductions in symptoms of fatigue in individuals

with musculoskeletal conditions. It will be of interest to determine whether other treatments typically used in the management of chronic pain, such as medication, heat/ice, laser, dry needling or electrical stimulation are also associated with reductions in fatigue.

Some degree of caution must be exercised in the interpretation of the study findings. First, data records were drawn from the clinical files of individuals referred to an occupational rehabilitation service. Only a minority of individuals with debilitating pain conditions are referred for occupational rehabilitation services. In addition, all participants were receiving long-term disability benefits. These sample characteristics necessarily have implications for the generalizability of findings. The modest sample size also limited the nature of analytic procedures that could be applied to the data. As well, it was not possible to examine the possible mediating or moderating role of other variables that could potentially influence the relation between pain and fatigue (i.e., type of medication, sleep, co-morbid health or mental 
health conditions). It is important to note that many participants in the present study were receiving other concurrent treatments such as medication or physical therapy. As such it is not possible to unambiguously attribute changes in pain and fatigue to the behavioural activation intervention.

In spite of these limitations, the results of the present study suggest that there is a temporal relation between symptoms of pain and fatigue in individuals with musculoskeletal conditions. The findings suggest that early treatment reductions in pain severity might contribute reductions in fatigue, and in turn, foster fuller participation in rehabilitation interventions. More research is needed to better understand the pathways underlying the temporal relation between pain and fatigue. More research attention also needs to be directed toward the development of more effective means of reducing fatigue in individuals with chronic musculoskeletal pain.

\section{Abbreviations}

CFI - Comparative Fit Index.

CLPM - Cross-lagged panel model.

FM - Fatigue Measure.

ICC - Intra-class correlation.

MPQ-PRI - McGill Pain Questionnaire - Pain Rating Index.

MPQ-SF - McGill Pain Questionnaire Short-Form.

RI-CLPM - Random-intercept cross-lagged panel model.

RMSEA - Root mean square error of approximation.

SRMR - Standardized root-mean-square residual.

TLI - Tucker Lewis Index.

\section{Declarations}

Ethics approval and consent to participate

This study was approved by the Institutional Review Board of McGill University.

\section{Consent for publication *}

Not applicable

\section{Availability of data and material *}


Data are available upon request from the MJL Sullivan.

\section{Competing interests}

The authors have no financial interest in the results of this research.

\section{Funding}

This research was supported by funds from the Canadian Institutes for Health Research (CIHR), the Canada Research Chairs program, and by a fellowship from the Astellas Foundation for Research on Metabolic Disorders and JSPS Overseas Research Fellowships (to K.Y.).

\section{Authors' contributions *}

All authors contributed to the writing and editing of the paper. KY conducted the statistical analyses. All authors approved the submitted version of the manuscript.

\section{Acknowledgements}

The authors thank Monique Gagnon and Frederic Chappe for their assistance in data collection and data base management.

\section{Sources of financial support}

This research was supported by funds from the Canadian Institutes for Health Research (CIHR), the Canada Research Chairs program, and by a fellowship from the Astellas Foundation for Research on Metabolic Disorders and JSPS Overseas Research Fellowships (to K.Y.).

\section{Disclosure}

The authors have no financial interest in the results of this research.

\section{Name of institutional review board}

This study was approved by the Institutional Review Board of McGill University.

\section{References}

1. Fishbain DA, Cole B, Cutler RB, Lewis J, Rosomoff HL, Fosomoff RS: Is pain fatiguing? A structured evidence-based review. Pain Med 2003, 4(1):51-62.

2. Lewis G, Wessely S: The epidemiology of fatigue: more questions than answers. J Epidemiol Community Health 1992, 46(2):92-97.

3. Murphy SL, Schepens Niemiec S, Lyden AK, Kratz AL: Pain, Fatigue, and Physical Activity in Osteoarthritis: The Moderating Effects of Pain- and Fatigue-Related Activity Interference. Arch Phys 
Med Rehabil 2016, 97(9 Suppl):S201-209.

4. Nikolaus S, Bode C, Taal E, van de Laar MA: Fatigue and factors related to fatigue in rheumatoid arthritis: a systematic review. Arthritis Care Res (Hoboken) 2013, 65(7):1128-1146.

5. Dailey DL, Frey Law LA, Vance CG, Rakel BA, Merriwether EN, Darghosian L, Golchha M, Geasland KM, Spitz R, Crofford LJ et al: Perceived function and physical performance are associated with pain and fatigue in women with fibromyalgia. Arthritis Res Ther 2016, 18:68.

6. Andrykowski MA, Donovan KA, Jacobsen PB: Magnitude and correlates of response shift in fatigue ratings in women undergoing adjuvant therapy for breast cancer. J Pain Symptom Manag 2009, 37(3):341-351.

7. Bansevicius D, Westgaard RH, Sjaastad OM: Tension-type headache: pain, fatigue, tension, and EMG responses to mental activation. Headache 1999, 39(6):417-425.

8. Feuerstein M, Carter RL, Papciak AS: A prospective analysis of stress and fatigue in recurrent low back pain. Pain 1987, 31(3):333-344.

9. Wolfe F, Hawley DJ, Wilson K: The prevalence and meaning of fatigue in rheumatic disease. J Rheumatol 1996, 23(8):1407-1417.

10. Van Damme S, Becker S, Van der Linden D: Tired of pain? Toward a better understanding of fatigue in chronic pain. Pain 2018, 159:7 - 10.

11. Siivola SM, Levoska S, Latvala K, Hoskio E, Vanharanta H, Keinanen-Kiukaanniemi S: Predictive factors for neck and shoulder pain: a longitudinal study in young adults. Spine (Phila Pa 1976) 2004, 29(15):1662-1669.

12. Halder SL, McBeth J, Silman AJ, Thompson DG, Macfarlane GJ: Psychosocial risk factors for the onset of abdominal pain. Results from a large prospective population-based study. Int J Epidemiol 2002, 31(6):1219-1225; discussion 1225-1216.

13. Dietvorst E, Hiemstra M, Hillegers MHJ, Keijsers L: Adolescent Perceptions of Parental Privacy Invasion and Adolescent Secrecy: An Illustration of Simpson's Paradox. Child Dev 2018, 89(6):20812090.

14. Hamaker EL, Kuiper RM, Grasman RP: A critique of the cross-lagged panel model. Psychol Methods 2015, 20(1):102-116.

15. Burckhardt CS, Jones KD: Effects of chronis widespread pain on the health status and quality of life of women after breast cancer surgery. Health Qual Life outcomes 2005, 3(1):30.

16. Hood A, Pulvers K, Carillo J, Merchant G, Thomas M: Positive traits linked to less pain through lower pain catastrophizing Pers Individ Dif 2012, 52(3):401-405.

17. Yamada K, Adams H, Ellis T, Clark R, Sully C, Sullivan MJL: Reductions in Fatigue Predict Occupational Reengagement in Individuals with Work-Related Musculoskeletal Disorders. J Occup Rehabil 2019.

18. Sullivan MJL, Adams H, Ellis T: A psychosocial risk-targeted intervention to reduce work disability: Development, evolution and implementation. Psychol Injury Law 2013, 6:1407 - 1415. 
19. Yamada K, Adams H, Ellis T, Clark R, Sully C, Sullivan MJL: Reductions in Fatigue Predict Occupational Reengagement in Individuals with Work-Related Musculoskeletal Disorders. J Occup Rehabil 2020, 30(1):135-145.

20. Falkenstrom F, Finkel S, Sandell R, Rubel JA, Holmqvist R: Dynamic models of individual change in psychotherapy process research. J Consult Clin Psychol 2017, 85(6):537-549.

21. Bentler PM, Yuan KH: Structural Equation Modeling with Small Samples: Test Statistics. Multivariate Behav Res 1999, 34(2):181-197.

22. Fishbain DA, Hall JA, Risser RC, Gonzales JS: Does pain cause the perception of fatigue in patients with chronic pain? Findings from studies for management of diabetic peripheral neuropathic pain with duloxetine. Pain Pract 2009, 9(5):354-362.

23. Jakobsson U: A literature review on fatigue among older people in pain: prevalence and predictors. Int J Older People Nurs 2006, 1(1):11-16.

24. Nicassio PM, Moxham EG, Schuman CE, Gevirtz RN: The contribution of pain, reported sleep quality, and depressive symptoms to fatigue in fibromyalgia. Pain 2002, 100(3):271-279.

25. Stone AA, Broderick JE, Porter LS, Kaell AT: The experience of rheumatoid arthritis pain and fatigue: examining momentary reports and correlates over one week. Arthritis Care Res 1997, 10(3):185-193.

26. Christie A, Dagfinrud H, Mowinckel P, Hagen KB: Variation in fatigue may be poorly explained by pain: results from a longitudinal, exploratory study. Rheumatol Int 2016, 36(2):279-282.

27. Lenaert B, Meulders A, van Heugten CM: Tired of pain or painfully tired? A reciprocal relationship between chronic pain and fatigue. Pain 2018, 159(6):1178-1179.

28. Olson K, Turner AR, Courneya KS, Field C, Man G, Cree M, Hanson J: Possible links between behavioral and physiological indices of tiredness, fatigue, and exhaustion in advanced cancer. Support Care Cancer 2008, 16(3):241-249.

29. Aili K, Andersson M, Bremander A, Haglund E, Larsson I, Bergman S: Sleep problems and fatigue as predictors for the onset of chronic widespread pain over a 5- and 18-year perspective. BMC Musculoskelet Disord 2018, 19(1):390.

30. Louati K, Berenbaum F: Fatigue in chronic inflammation - a link to pain pathways. Arthritis Res Ther 2015, 17:254.

31. Kwekkeboom KL, Tostrud L, Costanzo E, Coe CL, Serlin RC, Ward SE, Zhang Y: The Role of Inflammation in the Pain, Fatigue, and Sleep Disturbance Symptom Cluster in Advanced Cancer. $J$ Pain Symptom Manage 2018, 55(5):1286-1295.

32. Hodges PW, Danneels L: Changes in Structure and Function of the Back Muscles in Low Back Pain: Different Time Points, Observations, and Mechanisms. J Orthop Sports Phys Ther 2019, 49(6):464476.

33. Saligan LN, Lukkahatai N, Holder G, Walitt B, Machado-Vieira R: Lower brain-derived neurotrophic factor levels associated with worsening fatigue in prostate cancer patients during repeated stress from radiation therapy. World J Biol Psychiatry 2016, 17(8):608-614. 
34. Cordeiro LMS, Rabelo PCR, Moraes MM, Teixeira-Coelho F, Coimbra CC, Wanner SP, Soares DD:

Physical exercise-induced fatigue: the role of serotonergic and dopaminergic systems. Braz J Med Biol Res 2017, 50(12):e6432.

35. Lallukka T, Hiilamo A, Oakman J, Manty M, Pietilainen O, Rahkonen O, Kouvonen A, Halonen JI:

Recurrent pain and work disability: a record linkage study. Int Arch Occup Environ Health 2020, 93(4):421-432.

36. Snekkevik H, Eriksen HR, Tangen T, Chalder T, Reme SE: Fatigue and depression in sick-listed chronic low back pain patients. Pain Med 2014, 15(7):1163-1170.

37. Manning K, Kauffman BY, Rogers AH, Garey L, Zvolensky MJ: Fatigue severity and fatigue sensitivity: relations to anxiety, depression, pain catastrophizing, and pain severity among adults with severe fatigue and chronic low back pain. Behav Med 2020:1-9.

\section{Figures}

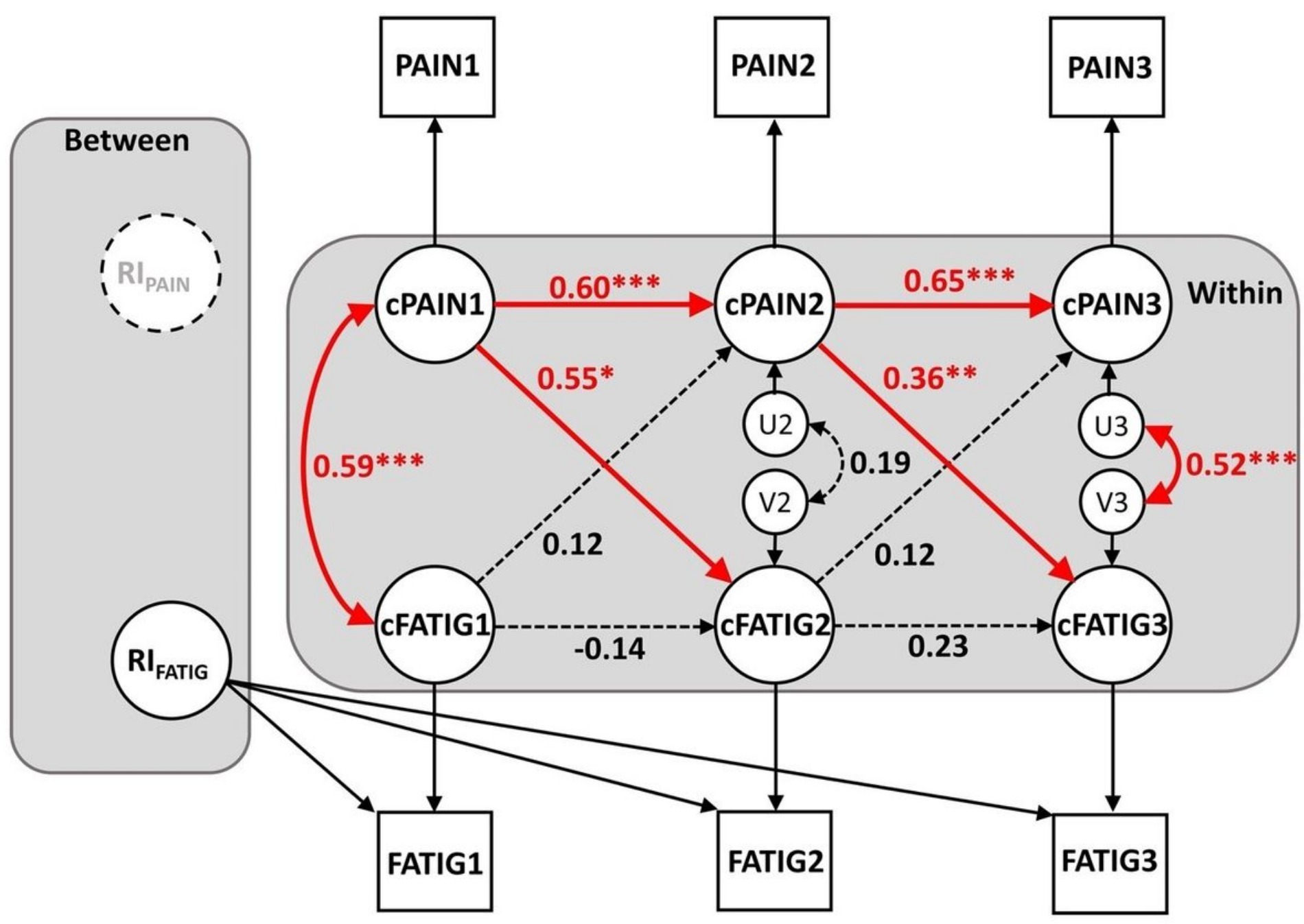

Figure 1 
Random Intercepts Cross-Lagged Panel Model Linking Pain and Fatigue. Note: N=104. Model fit indices: comparative fit index $(\mathrm{CFI})=1.00$, Tucker-Levis index $(\mathrm{TLI})=1.00$, root mean square error of approximation (RMSEA) < 0.001 (90\% confidence interval: 0.00-0.15), standardized root-mean-square residual $(S R M R)=0.03$. 